\title{
Enhanced 3D-Visualization of Intracranial Aneurysms Involving the Skull Base
}

\author{
F. Vega Higuera ${ }^{1,2}$, N. Sauber ${ }^{2}$, B. Tomandl ${ }^{3}$, \\ C. Nimsky ${ }^{1}$, G. Greiner ${ }^{2}$, and P. Hastreiter ${ }^{1,2}$ \\ 1 Neurocenter, Dept. of Neurosurgery, University of Erlangen-Nuremberg, Germany \\ vega@neurozentrum.imed.uni-erlangen.de \\ 2 Computer Graphics Group, University of Erlangen-Nuremberg \\ 3 Div. of Neuroradiology, Dept. of Neurosurgery, University of Erlangen-Nuremberg
}

\begin{abstract}
Direct volume visualization has recently gained importance as a tool for the analysis of intracranial aneurysms focused on therapy planning. CT-angiography (CTA) intensities are mapped to color and opacity values by so called one-dimensional transfer functions. In this work, we introduce 3D-visualization of intracranial aneurysms making use of transfer functions based on measured values and gradient magnitudes extracted from the CTA data. Furthermore, we present a tool for the creation of $2 \mathrm{D}$ transfer functions in the clinical environment. The visualization application runs on standard PCs equipped with modern 3D graphics cards. Evaluations were carried out on 17 clinical cases from our archive. Clear improvements with respect to standard volume visualization were observed especially in the area of the skull base, where the arteries are difficult to separate from the bone. Effective separation of skull and arteries was achieved even for cases where the critical vascular structures were embedded in osseous tissue.
\end{abstract}

Keywords: 3D-Visualization, Transfer Function, Aneurysm, Skull Base

\section{Introduction}

Direct volume visualization has proven its importance for therapy planning of intracranial aneurysms [2,3]. CTA data provides the basis to create renderings of vascular structures through the mapping of intensities to color and opacity values by the so called transfer function (TF). Due to the characteristics of the source data and the occurring partial volume effect, the mentioned standard TF produce good quality results only if the vessels are anatomically well separated from the skull base. Otherwise, the similarity between measured intensities for osseous tissues and vascular structures filled with contrast agent leads to incorrect or non-clear 3D-visualizations of the lesions.

Moreover, intracranial aneurysms often occur close to the skull base 9], which makes it difficult or even impossible to achieve a clear volume visualization based on standard TF. A possibility to overcome this problem could be subtraction-CTA, however, it implies double radiation exposure for the patient. Another alternative could be to extract further features from the data in order to 
create multidimensional TF. As a result of that, the increased flexibility allows clearer representation of the structures of interest.

Transfer functions based on intensity and gradient magnitude values were first introduced by Levoy [6]. Recently, Kniss and Kindlmann [5] proposed a tool that permits interactive manipulation of multidimensional TFs for volume visualization. In addition to that, we introduce $2 \mathrm{D}$ TFs to the clinical problem of aneurysms close to the skull base. We propose the use of this strategy for therapy planning, by providing clinically suited tools for the creation and interactive manipulation of 2D TFs. The proposed approach does not require any special hardware for volume visualization and it was implemented on PCs equipped with 3D graphics cards.

\section{2D Transfer Functions}

Direct volume visualization is based on the solution of the radiance equation for light transport [6]. Physical quantities describing the light emission and absorption are assigned to every voxel. This process is usually done by means of transfer functions that map data values to colors (emission) and opacities (absorption). The standard 1D TF approach is only based on the mapping of measured intensities.
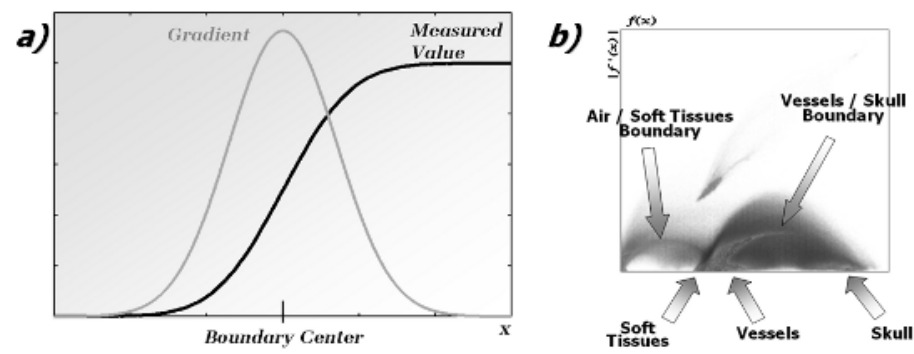

Fig. 1. a) Measured value and gradient at the boundary. The measured value at the boundary (shown black) is modeled by the erf function. b) 2D Histogram from a CTA dataset.

2D TFs as proposed by Levoy [6] were aimed to produce isosurfaces from volume data. As a drawback of this strategy, previous knowledge about the characteristics of the data is required in order to achieve the desired results. Kindlmann [4] proposed an approach for the semi-automatic creation of 2D TFs. The main idea focuses on the creation of TFs that put emphasis on material boundaries. A boundary model represented by the erf function (see figure 11a) is defined and the behavior of the gradient at the boundary is analyzed. The erf function corresponds to a 1-dimensional CT-scan representation of an ideal boundary. Figure 1. a shows the shape of the measured value and the gradient function around the boundary center. It is easy to see how the maximum gradient value is reached at the middle point of the boundary as one might expect. 
This idea is extended to volume data where the intensity functions are 3D. Since it can be assumed that ideal boundaries are recreated by the CT-scan according to the mentioned boundary model, one can expect the same kind of behavior from the volume data. We have taken a slightly simplified approach in comparison to the 3D histogram from Kindlmann, in order to obtain information about boundaries within the source data. The gradient magnitude is extracted for every voxel in the dataset. Then, $2 \mathrm{D}$ histogram is computed where the $\mathrm{x}$-axis corresponds to the measured intensity and the $\mathrm{y}$-axis corresponds to the gradient magnitude. The obtained data is plotted as a $2 \mathrm{D}$ plane assigning gray values according to the number of occurrences for each coordinate. The final result is represented in figure 1 b. Patterns can be observed, where the characteristic curves for the gradient function become evident. Now it can be assumed that for every arc shown in the $2 \mathrm{D}$ histogram, there is a boundary in the volume data. Moreover, the average location of the boundary center is indicated by the intensity that takes the highest value in the gradient function. Transfer functions have to be shaped following the information available in the $2 \mathrm{D}$ histogram.

Kniss et al. [5] proposed an user interface for the creation of multidimensional TF through interactive manipulation of widgets. This strategy, which is based on the works from Levoy and Kindlmann, produced renderings of good quality for CT-data. Even so, this approach requires a great deal of expertise about multidimensional TF [7]. In this work, we produced TFs that yield to volume renderings where vascular structures are clearly identifiable, even if these are located next to the skull base. This was achieved by providing the adequate tools for the clinical use of $2 \mathrm{D} \mathrm{TF}$ for direct volume visualization of CTA data.

\section{Transfer Function Editor}

As already mentioned, TFs have to be shaped according to the boundaries contained within the dataset. In order to simplify this task, a TF editor which provides tools for rapid creation of 3D-visualization of CTA data was developed.

Information about the tissues and boundaries contained in the data is presented in the form of the 2D histogram that has been computed at loading time. The histogram is applied as background for the work area which is used for creation and adjustment of transfer functions (see figure 2). The investigator can paint the TF as a 2D image. Editing is carried out using the histogram as guidance parameter, always providing information about the tissues and their boundaries. Moreover, since tissues and their boundaries are clearly identified in the histogram, it is possible to apply colors and opacities to each structure individually. One can for example, put emphasis on the vessel side of the vessel/skull boundary and in this manner reduce the artifacts produced by partial volume effects.

Operation of the editor is based on the boundary function widget that we developed. Kindlmann [4] proposed semi-automatic creation of TFs based on the intensity and gradient magnitude producing promising results with reference CT-datasets. Nevertheless, experiments with this approach proved it to be 
less effective with CTA datasets. One of the determining factors is the required fine tuning of threshold parameters as a preprocessing step. These parameters have to be adjusted according to the individual characteristics of each dataset. Misadjustment produces shifting of the transfer function and as a result, target structures become unrecognizable. We propose the use of a widget that replicates the shape of the gradient function around the ideal boundary and makes it possible to create boundary functions adjusted by the user interactively.

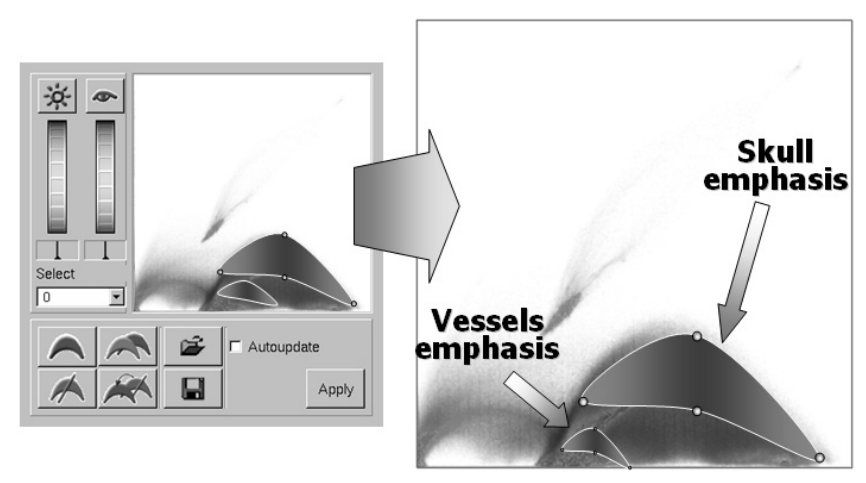

Fig. 2. 2D transfer function created in the editor. Two boundary function widgets are shown; one put emphasis on vessels while the second on osseous tissues.

As discussed before, around the boundary center the gradient function takes the shape of a Gaussian curve. In order to provide smooth real-time interaction, the function is modeled using quadratic Bézier curves whose control points can be shifted in real time (see figure 2). Since the widget replicates the shape of the boundaries that are shown in the $2 \mathrm{D}$ histogram, it is easy to determine were to place it initially and then to adjust it.

Parameters for the widget like boundary opacity and width can be adjusted with data sliders. Colors and opacities are easy to assign with standard color dialogs and results are available in real time. Global controls for intensity and opacity are provided permitting fine tuning of the final 3D-visualization. The created TFs can be saved to use them as templates for other datasets. Since the behavior of the gradient function around the boundary is consistent for every dataset, only fine tuning of the templates is necessary.

Integration into Volume Rendering: 3D-visualization is performed making use of the pixel shader unit available on PC graphics cards [8. A 3D RGBA texture with the same size as the CTA dataset is used as previous texture for the dependent texture operation; intensity values and gradient magnitudes extracted from the source data are stored as the $\mathrm{R}$ and $\mathrm{A}$ components of every texel. The $2 \mathrm{D} \mathrm{TF}$ is stored as a $256 \times 256$ texture which is used as dependent texture. As a result, the RGBA output of the shader unit contains the mapping of the source data to color and opacity values made by the $2 \mathrm{D} \mathrm{TF}$. In addition to this, per-voxel gradients are stored and used to compute per-pixel Phong illumination 
making use of the register combiners. The output values of the shader unit are then used for texturing the parallel planes that make up the visualization as proposed by Cabral et al. 1].

Since the gradient and its magnitude are computed only once at loading time and the TF is applied by the graphics hardware, the process of adjusting the transfer function is interactive and it is supported by real-time feedback.

\section{Results and Discussion}

The presented strategy have been validated with a set of 17 clinical cases. The source CTA data was obtained with a spiral-CT scanner. The reconstructed voxel size of the datasets is $0.23 \times 0.23 \times 0.5 \mathrm{~mm}^{3}$. The applied datasets consisted of images with a $512 \times 512$ matrix and 70-130 slices. In order to focus on the lesion, sub-volumes containing the area of interest for each dataset were created using a resolution of $256 \times 256 \times 64$ voxels. No preprocessing such as explicit segmentation was necessary.

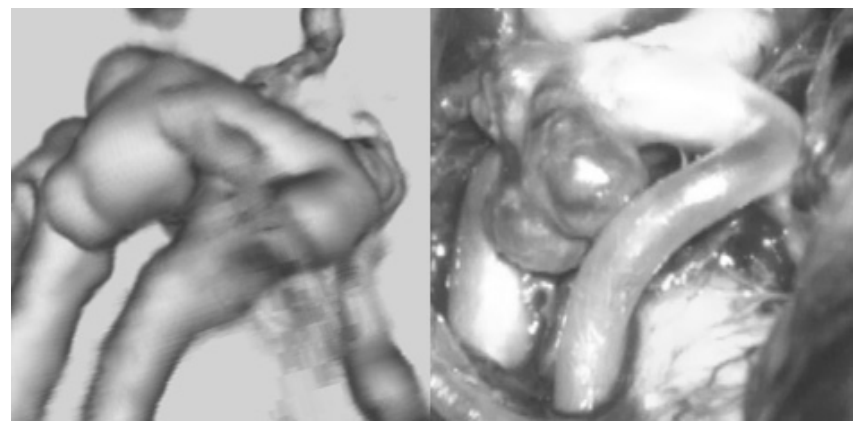

Fig. 3. 3D rendering of intracranial aneurysm (left) and intraoperative image (right).

The presented approach was evaluated in comparison to intraoperative observations (see figure 3) and 3D renderings obtained with 1D TFs [2]. On a first stage, different TFs aimed to achieve clear delineation of vascular structures and osseous tissues removal were created. Once these templates were available, the fine adjustments for each particular dataset required a short period of time.

Especially in areas with intensive presence of partial volume effects, the results obtained with the suggested technique led to improved visualizations when compared to using 1D TFs. The case presented in figure 4 shows an intracranial aneurysm located at the skull base and under osseous tissue. It can be appreciated how the rendering produced with $1 \mathrm{D} \mathrm{TF}$ fails to clearly separate the aneurysm from the skull at the upper side of the lesion, while $2 \mathrm{D}$ TF visualization is able to faithfully delineate the different tissues. Also, vessels running next and through the skull are better represented. 

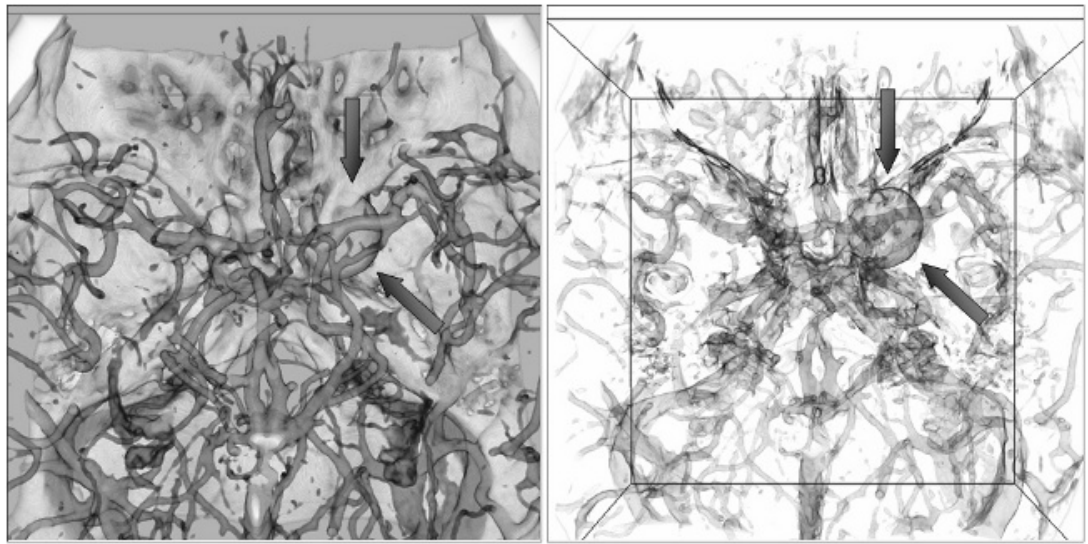

Fig. 4. 3D-visualization with $1 \mathrm{D}$ (left) and 2D (right) TFs. An aneurysm of the right internal carotid artery within the skull base is shown (arrows).

3D-visualizations of the sub-volume containing the area of interest in this case show the value of the presented approach (see figure 51). In this particular example, the parent vessels are located next to the skull base. Renderings obtained with 1D TFs are not able to clearly delineate the target structure since it is located behind osseous tissue. 2D transfer functions allow to suppress the skull providing a clear view to the areas of interest, even if they are hidden by osseous tissue.

A second example shows the value of the approach for cases with strong partial volume effects (see figure 6). This aneurysm is embedded in the skull base. Again, the parent vessel is located underneath osseous tissue. Artifacts produced during the CT-scan, which affect the aneurysm area, represent an additional difficulty. The achieved results are similar to those obtained with explicit segmentation techniques. Although partial volume effects prevent the TF from completely suppressing the skull, areas of interest are clearly delineated. This information help to plan therapy in a more efficient and precise way.

\section{Conclusion and Perspectives}

The presented work demonstrates the possible value of direct volume rendering making use of 2D transfer functions for (surgical) therapy planning, since precise $3 \mathrm{D}$-visualization of aneurysms located close to the skull base is obtained.

The developed tools provide the frame-work for easy application of this strategy. Adjustment of a transfer function template for each particular case takes less than 10 minutes per patient. Further developments will aim at the automation of this process. The main advantage of the presented approach over 1D transfer functions becomes evident when partial volume effects prevent a clear delineation of lesions. On the other hand, interactive frame rates were achieved 

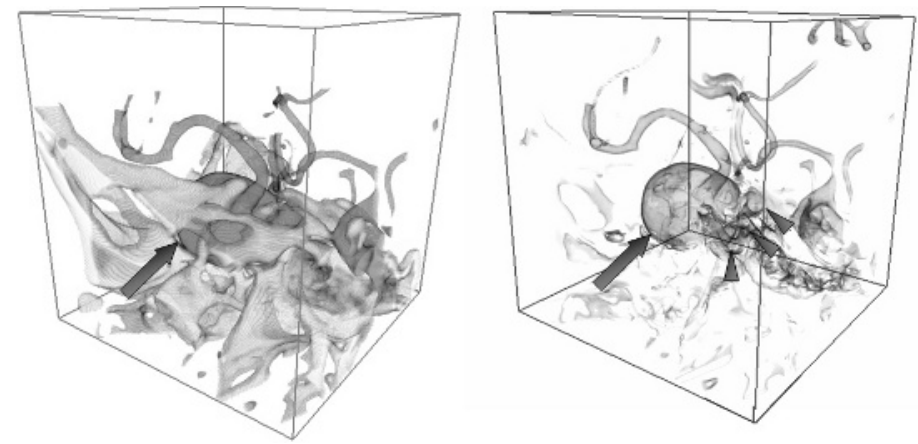

Fig. 5. The left image shows results produced with $1 \mathrm{D}$ TF while the right image represents the same visualization with 2D TF. The parent arteries (arrow heads) of this aneurysm (arrows) of the right internal carotid artery are more clearly delineated with the suggested approach.
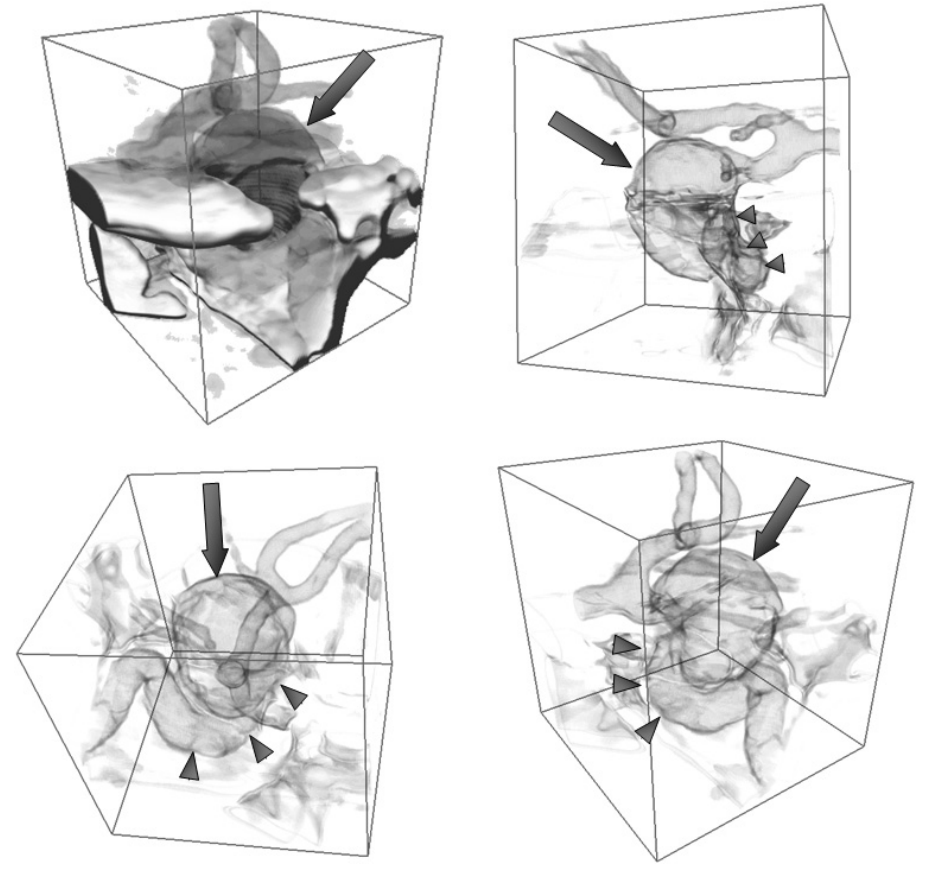

Fig. 6. 3D-Visualization based on 2D TF: osseous tissue removal and delineation of important structures of an aneurysm (arrows) of the right internal carotid artery involving the skull base. The course of the internal carotid artery (arrow heads) within the skull base is clearly visible. 
making use of PCs equipped with consumer graphics cards. Thereby, the process of creating the transfer functions is supported by real-time feedback, and the presented technique is made affordable for standard clinical application.

Altogether, the suggested approach allows a clear and precise separation of anatomical structures with reduced differences in data values and generally makes direct volume visualization more useful for therapy planning, even for aneurysms within the skull base.

Acknowledgments. This work was funded by Deutsche Forschungsgemeinschaft in the context of the project GRK 244.

\section{References}

1. B. Cabral, N. Cam, and J. Foran. Accelerated volume rendering and tomographic reconstruction using texture mapping hardware. In Proceedings of the 1994 symposium on Volume visualization, pages 91-98. ACM Press, 1994.

2. P. Hastreiter, C. Rezk-Salama, B. Tomandl, K. Eberhart, and T. Ertl. Fast Analysis of Intracranial Aneurysms based on Interactive Direct Volume Rendering and CTAngiography. In Proceedings Medical Image Computing and Computer Assisted Intervention. MICCAI, Springer, 1998.

3. S. Iserhardt-Bauer, P. Hastreiter, B. Tomandl, N. Köstner, M. Schempershofe, U. Nissen, and T. Ertl. Standardized Analysis of Intracranial Aneurysms using Digital Video Sequences. In Proceedings Medical Image Computing and Computer Assisted Intervention, pages 411-418. MICCAI, Springer, 2002.

4. G. Kindlmann and J. W. Durkin. Semi-automatic generation of transfer functions for direct volume rendering. In Proceedings of the 1998 IEEE symposium on Volume visualization, pages 79-86. ACM Press, 1998.

5. J. Kniss, G. Kindlmann, and C. Hansen. Interactive volume rendering using multidimensional transfer functions and direct manipulation widgets. In Proceedings of the conference on Visualization 2001, pages 255-262. IEEE Press, 2001.

6. M. Levoy. Display of surfaces from volume data. IEEE Computer Graphics and Applications, 8(3):29-37, 1988.

7. H. Pfister, B. Lorensen, C. Bajaj, G. Kindlmann, W. Schroeder, L. S. Avila, K. Martin, R. Machiraju, and J. Lee. The transfer function bake-off. IEEE Computer Graphics and Applications, 21(3):16-22, 2001.

8. C. Rezk-Salama, K. Engel, M. Bauer, G. Greiner, and T. Ertl. Interactive volume on standard pc graphics hardware using multi-textures and multi-stage rasterization. In Proceedings SIGGRAPH/EUROGRAPHICS workshop on Graphics hardware, pages 109-118. ACM Press, 2000.

9. N. Young, N. Dorshc, R. Kingston, G. Markson, and J. McMahon. Intracranial aneurysms: evaluation in 200 patients with spiral ct-angiography. European Radiology, 11(1):123-130, 2001. 